\title{
Energy Efficient Data Transmission Scheme for Wireless Sensor Network using DSC-MIMO
}

\author{
Krati Varshney \\ Dept. ECE, Krishna \\ Institute of Engineering \& \\ Technology, Ghaziabad, \\ UP, INDIA
}

\author{
Manish Kumar \\ Singh \\ Dept. ECE, Krishna \\ Institute of Engineering \& \\ Technology, Ghaziabad, \\ UP, INDIA
}

\author{
Vibhav Kumar \\ Sachan, PhD \\ Dept. ECE, Krishna \\ Institute of Engineering \& \\ Technology, Ghaziabad, \\ UP, INDIA
}

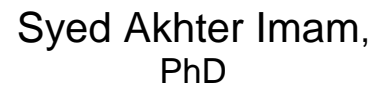

Dept. ECE, Jamia Millia

Islamia, New Delhi

\begin{abstract}
A Wireless sensor network is made up of small sensor devices which can communicate with each other and that are Battery operated. The Energy resources are limited and battery replacement is not easy work. There are so many application of sensor network such as Target tracking, Remote monitoring, waste water monitoring, data logging, natural disaster prevention, land slide detection, etc. Because energy resources are limited so there is a need of energy saving mechanisms which can efficiently reduce power level. In this paper we will analyze energy consumption of distributed source coding MIMO system. There are three domains in sensor network where power consumption has occurred that are Sensing, communication and data processing.

In this paper, we will discuss about DSC-MIMO methods to reduce power consumption and increase energy efficiency and will compare with traditional SISO and MIMO system. DSC (Distributed Source Coding) schemes are used to compress data of sensor nodes which is correlated. By this method we can gain high coding efficiency and MIMO is effectively reducing energy consumption.
\end{abstract}

\section{Keywords}

WSN (Wireless sensor network), DSC (Distributed source coding), MIMO (Multiple input Multiple output), SISO (Single input Single output), Power consumption.

\section{INTRODUCTION}

Distributed source coding is used to compress the correlated data of sensor nodes without intercommunication between the nodes. So, we can reduce Bandwidth and energy for the many WSN applications. Distributed source coding can be done in two manner First, the senor can communicate with each other through an inter sensor network, or second, they could avoid doing this. The first option gives an extra unwanted overload of creating an additional network and require more processing in each node. The point of source coding in sensor network is accurately the opposite; to reduce the handling amount and thus the energy consumption. This could be avoided using the second scheme.

Now the major thing that comes into mind with this choice is that without the idea of the other sensors data, how can the dynamic sensor compress anything at all? Well, as Slepian and Wolf showed that the data can be compressed as much as with knowledge of what the other sensors send. This is known as the Slepian-Wolf theorem and fairness is of course only applicable in theory. It is derived asymptotically and based on random binning principles. But it may work as a practical objective and give us good measures on how well we are doing. In multiple input $\&$ multiple output system there are multiple antennas at receiver and transmitter side. Performance of the system can be improved by MIMO as increase data rates by spatial multiplexing or through diversity. MIMO systems are highly energy efficient because transmission energy is less than SIS0 system.

MIMO technology can increase the channel capacity because of the multiplexing gain, and the communication reliability because of the diversity gain. In another words, for the same bit-error-rate, a MIMO system uses less transmitting energy than outdated single-input single-output (SISO) mode. It is this idea that kinds MIMO technology a possible option for data transmission in wireless sensor networks. Considering the simple limitation of sensors physical size, it is impossible to install multiple antennas in one sensor node. So, the MIMO transmission in wireless sensor networks must be operated in a supportive way, which means multiple sensor nodes with single-antenna in each of them, not multiple antennas, form this MIMO link.

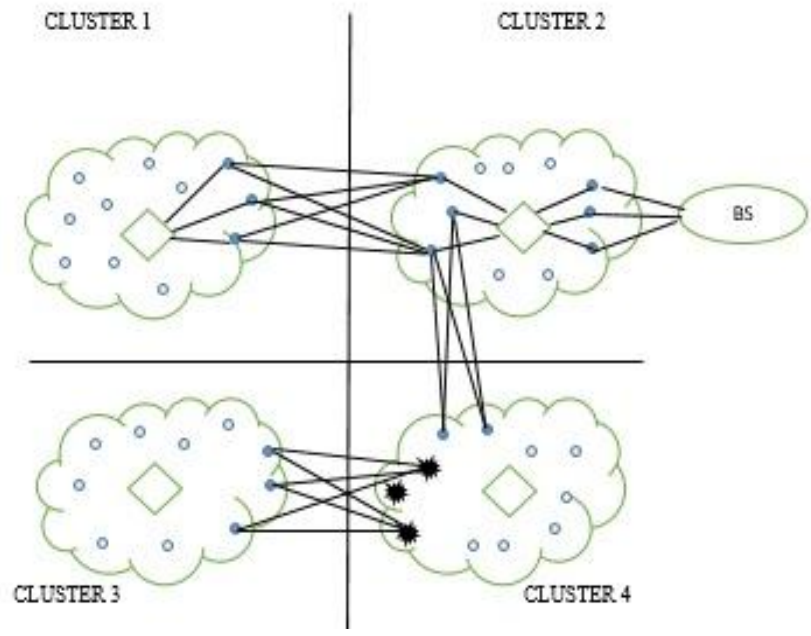

Fig.1: Virtual MIMO Wireless Sensor Network

In multiple input $\&$ multiple output system there are multiple antennas at receiver and transmitter side. Performance of the system can be enhanced by MIMO as increase data rates by spatial multiplexing or through diversity. MIMO systems are extremely energy efficient because transmission energy is less than SIS0 system.

MIMO technology can rise the channel capacity because of the multiplexing gain, and the communication reliability because of the diversity gain. In another words, for the similar bit-error-rate, a MIMO system uses less transmitting energy than traditional single-input single-output (SISO) mode. It is 
this idea that makes MIMO technology a likely option for data transmission in wireless sensor networks. Considering the strict limitation of sensors' physical size, it is impossible to fit multiple antennas in one sensor node. So, the MIMO transmission in wireless sensor networks must be operated in a cooperative way, which means multiple sensor nodes with single-antenna in each of them, not multiple antennas, form this MIMO link. There are also some extra systems derived from MIMO, which are known as SIMO, MISO. Diagrams illustrating SISO, SIMO, MISO and MIMO are shown in Figure:
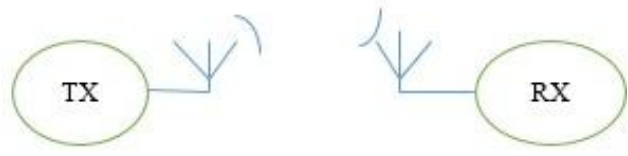

Fig 2(a): SISO
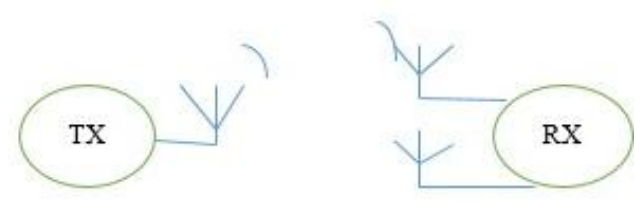

Fig 2(b): SIMO
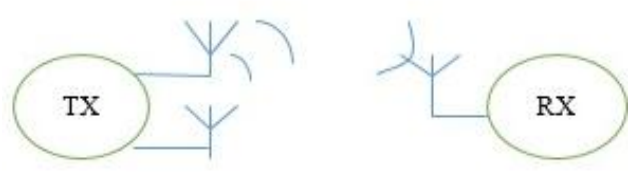

Fig 2(c): MISO

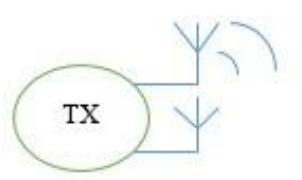

Fig 2(d): MIMO

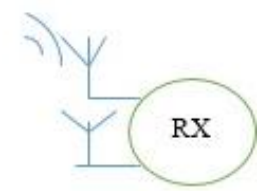

Fig.2: Various Transmission Modes of Wireless Sensor Network

\section{RELATED WORK}

Firstly, in Cui et al. (2004), the authors propose MIMO for WSNs, where MIMO is based on Alamouti diversity schemes and it is extended to individual single antenna group nodes. The array nodes work together with each other to tradition multiple antenna transmitters or receivers. By receiving and sending information jointly, a lot of energy can be saved. MIMO system outdoes SISO (Single Input Single Output) after a certain distance. By maintaining the proper constellation (bits per symbol) size, MIMO can outperform SISO in case of long haul distance. The author comprehensive the idea to implement virtual MIMO where each antenna on the transmitter side is used as the antenna array for MIMO communication.

In Cui et al. (2005), the author's integrate multiple layers for co-operative transmission. By scheming routing and link scheduling in upper layers, MIMO can reduce energy ingestion with improving end-to-end performance. In this work, long haul communication for base station is observed by $2 * 2$ MIMO without any local information exchange. In del Coso et al. (2006), the optimal time management and power budget distribution for virtual MIMO is proposed. Their analysis shows that virtual MIMO works like actual MIMO for low signal to noise ratio. The outcomes show that the energy convertible is increased with the increase of hops. According to Chen et al. (2005b), the optimized number of nodes in a cluster and optimized number of hops save energy for virtual MIMO communication.

Training overhead and orthogonal STBC have been examined in their MIMO architecture to support channel estimation and multiple antennas. In their analysis, the same cluster of nodes are used as transmitters and receivers for relay, which reasons the early death of network. In Jayaweera (2004), the authors include the training codes for fading channels and their analytical results show that the energy consumption of MIMO is lower as compared to SISO. In Jayaweera (2005), data assembly are considered without any energy constraint and multiple antennas are integrated on these nodes.

Channel path loss and periodic codes for channel estimate are incorporated to approximation total energy consumption. In Jayaweera (2006), the energy consumption is considered for 2x2 MIMO for the additional overhead symbols for multiple transmitter and receiver antennas. Although more training overhead is needed for multiple transmitters, the analytical model shows that the energy consumption is low as compared to SISO, when transmission distance, transmission rate, and time period are carefully sustained. In Jayaweera, the receiver nodes cooperate instead of transmitter nodes; the transmission time should be adaptive to attain lower energy consumption as compared to SISO.

In Yuan and He (2006a), a cross layer architecture is planned, where multiple senders transmit data to single receiver of the adjacent cluster. The choice of appropriate set of transmitters can save significant energy for MIMO. In Yuan and $\mathrm{He}$ (2006b), the authors study both the inter cluster and intra cluster communication energy to select the 2 suitable supportive transmitter nodes. In the energy consumption model, AGWN and Rayleigh Fading channels have been considered for intra-cluster and inter-cluster communication respectively. In Zhang and Dai (2007), space-time block coding and spatial multiplexing code on optimal transmission strategy is defined.

The scheme can decrease the critical replacement for virtual MIMO. The authors have also studied the switching parameters of coding system for energy adaptable high rate data transmission in MIMO. In Chen et al. (2005a), the energy consumption for virtual MIMO communication in multi hop network is analyzed. The proposed protocol selects the nodes and provides the routing path for efficient MIMO communication. Different MIMO combinations are considered for full cooperative and half cooperative transmissions in Liu and Xiaohua Li (2005). In Qing-hua et al. (2007a), cooperative nodes are selected for MIMO communication. Fixed rate data are considered for communication between clusters. The energy model justifies the efficiency of virtual MIMO communication for sparse sensor network.

The researchers have made a lot of admirable virtual MIMO communication scheme. Cui et al. anticipated a model of virtual MIMO communication in single-hop wireless sensor networks with Alamouti coding [2].They analyzed the energy consumption of the network and the data propagation delay and then compared the energy consumption of virtual MIMO with SISO's in the same bit error rate requirement. Their research shown that the virtual MIMO was more suitable than SISO in the long-distance data transmission according to 
energy efficiency and data transfer delay. Jayaweera examined the effect of the modulation constellation size, the transmission distance, the increasing control overhead of the training sequence, and delay performance of virtual MIMO in wireless sensor networks [3].

\section{DATA COMPRESSION USING SOURCE CODING}

Distributed source coding is probable when there is correlation between a set of sources. This is accurately the case in typical sensor networks, where the correlation often is great between neighboring nodes. Each distinct node is compressing their data based on observations done by other sensors and not only on its individual localized data, hence the term distributed. To exploit this correlation and eliminate the redundancy, each node has to know approximately about what the other sensors send. Imagine you have two sensor nodes and you want to compress the data they communicate as much as possible within a fidelity criterion. Sensor data sequence $\mathrm{X}$ is input into the encoder which compresses $\mathrm{X}$ based on the correlation distribution between $\mathrm{X}$ and $\mathrm{Y}$. $\mathrm{Y}$ is sent uncompressed to the decoder as side information as demonstrated in Fig. 2. Now the purpose of the joint decoder is to estimate $\mathrm{X}$ based on the received data and the side information Y. The more correlated the sources are, the more sure we can be that the chosen estimate is the correct one, or the more we can compress X.

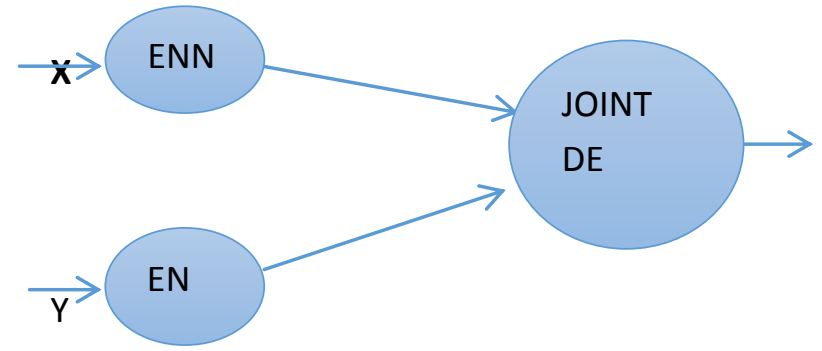

Fig.3: Lossless Distribution source coding

The proof of the Slepian-Wolf theorem is formed on random binning. Binning is a key concept in DSC and refers to separating the space of all possible results of a random source into disjoint subsets or bins. Examples explaining the binning process are given in Box 1. The achievability of Slepian-Wolf coding was generalized by Cover [10] to arbitrary ergodic processes, countably infinite alphabets, and arbitrary number of correlated sources.

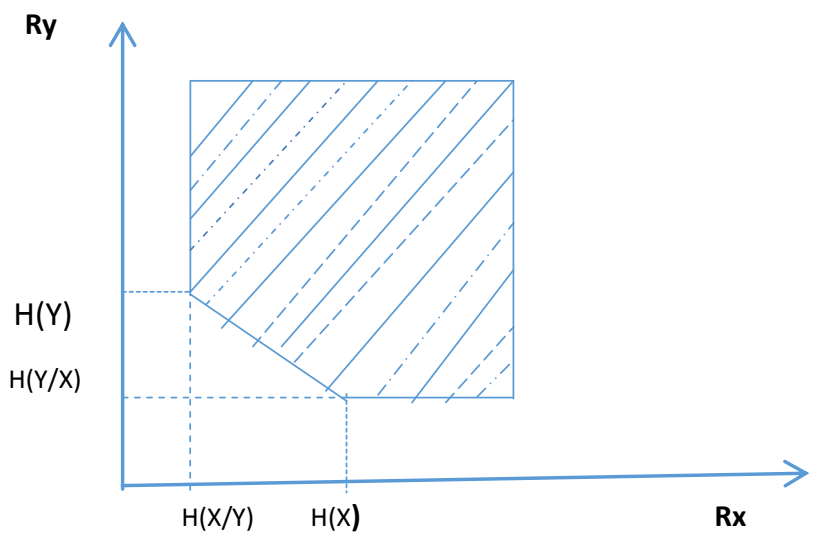

Fig.4: Rate region for Slepian-Wolf coding (two sources).

$$
\begin{aligned}
& R_{X} \geq H(X), \\
& R_{Y} \geq H(Y), \\
& R_{X}+R_{Y} \geq H(X, Y),
\end{aligned}
$$

The corner points may be extended by the asymmetric coding scheme. All the points on the line $\mathrm{H}(\mathrm{X}, \mathrm{Y})$ is available by either time sharing or by symmetric coding. Time sharing is done by letting one of the sources act as side information a part of the time and the other source the rest.

\section{RATE-DISTORTION THEORY}

When we consider equivalent sources, it is no longer possible to decode these losslessly because of the imperfect capacity of an accurate channel. Instead of having the unrestrained distortion from the channel we can introduce a certain controlled degradation or distortion to the signal before transmission. The more noise on the obtainable channel, the more we have to increase this distortion, or in other words: reduce the source rate.

$$
\begin{aligned}
& R(D) \\
& =p(\hat{x} \mid x): \sum_{(x, \hat{x})} \min _{p(x) p(\hat{x} \mid x) d(x, \hat{x}) \leq D} I(X ; \hat{X}),
\end{aligned}
$$

Recently some practical design methods have been proposed from the concept of channel coding. A practical DSC scheme called DISCUS (Distributed Source Coding Using Syndromes) was introduced in [4]. In DISCUS, instead of sending the code word, the syndrome of the code word coset is sent and the receiver decodes by searching in the given coset and selecting code word that is closest to side information. DISCUS based on LDPC codes was also addressed in [5] and [6]. LDPC codes seem to work better than turbo codes [7]. The development of distributed source coding of non-binary sources using LDPC codes proposed in [8]. The results demonstrate that the non-binary compression scheme achieves a very low bit error rate.

\section{DATA TRANSMISSION SCHEME}

A multiple-input multiple-output (MIMO) system contains of multiple antennas at the receiver and transmitter. These multiple antennas can be used to recover the performance of the system through spatial diversity or increase the data rates by spatial multiplexing. One can also use some of the antennas for diversity and some for spatial multiplexing. The number used for diversity and spatial multiplexing depends on the application. Zheng and Tse [1] provide an optimal tradeoff curve for multiplexing and diversity in the high SNR system.

MIMO systems can support higher data rates at the same transmission control and bit-error-rate (BER) requirements i.e. for the same throughput requirement, MIMO systems require less transmission energy. Hence it is attractive to believe that MIMO systems are more energy efficient than single-input single-output (SISO) systems. However the circuit energy consumption of a MIMO system is more than for a SISO system as it has numerous RF chains and requires more signal processing. Numerous studies on the energy efficiency of MIMO systems have been done.

We also assume that the system is uncoded. Hence, no error correction code (ECC) blocks are contained. The procedure 
used here can be extended to include those blocks in future research work. $M_{t}$ And $M_{r}$ are the numbers of transmitter and receiver antennas, individually, and we assume that the frequency synthesizer (LO) is joint among all the antenna paths. For the SISO case, we have $M_{t}=1 \& M_{r}=1$.

\section{EFFICIENT CODING SCHEME}

In this regard, error control coding (ECC) is an effective technique used to increase link reliability and to reduce the required transmitted power. In this context, the choice of energy efficient ECC with a suitable modulation scheme is a crucial task at the link and physical layer of wireless sensor networks to improve their lifetime. Since the WSNs are energy constraint in nature, both the probability of Bit error rate (BER) and power consumption have to be taken into account. A performance analysis of error control coding schemes referring to BPSK modulation through a Gaussian channel (AWGN) is presented in this paper. Our results show that the RS $(255,165)$ outperforms other ECC schemes and can be the optimal choice for wireless sensor network environment.

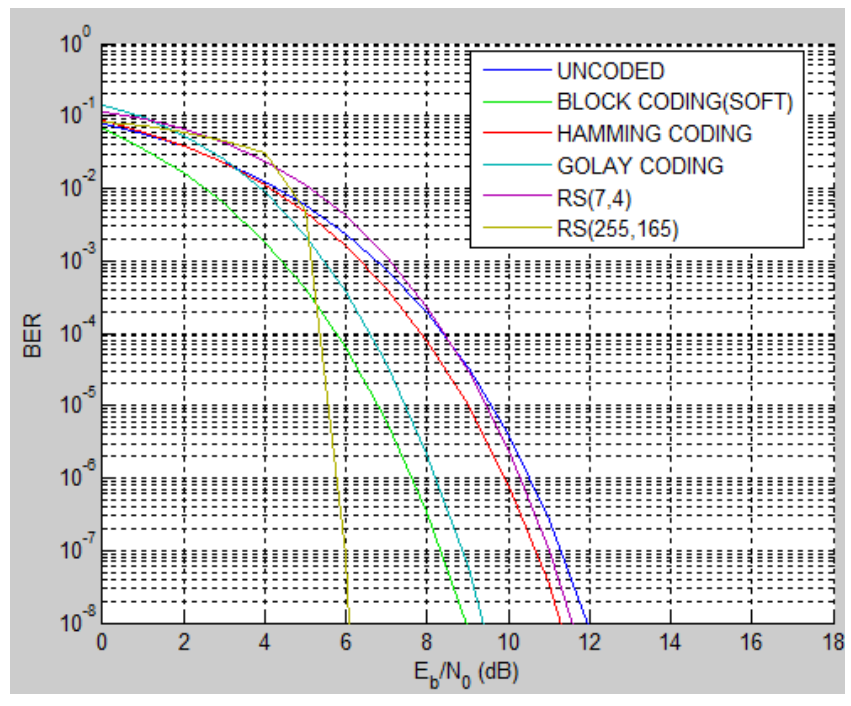

Fig.5: Effective Modulation Scheme

\section{SYSTEM MODEL}

The total average power consumption along the signal path can be divided into two main parts: The power consumption of all the power amplifiers $\mathrm{P}_{\mathrm{PA}}$ and the power consumption of all other circuit blocks.

The amplifier power consumption can be approximated as

$$
P_{P A}(d)=(1+\alpha) P_{\text {out }}(d),
$$

Where Pout is the transmit power, $\alpha=\xi / \eta-1$ with $\eta$ being the drain efficiency of the RF power amplifier and $\xi$ being the peak-to-average ratio (PAR), which depends on the modulation scheme and the constellation size.

Throughout this paper, as in [9-11], we assume M-QAM systems, so that

$$
\xi=3(\mathrm{M}-2 \sqrt{M}+1) /(\mathrm{M}-1),(6)
$$

The transmit power out $\mathrm{P}$ can be calculated as

$$
P_{\text {out }}(d)=\overline{E_{b}} R_{b} \frac{(4 \pi)^{2} d^{k} M_{l} N_{f}}{G_{t} G_{r} \lambda^{2}},
$$

where $d$ is the transmission distance, $k$ is the channel path loss exponent, $G_{t}$ and $G_{r}$ are the transmitter and receiver antenna gains, respectively, $\lambda$ is the carrier wavelength, $M_{l}$ is the link margin for compensating the hardware process variations and other additive background noise or interference, $N_{f}$ is the receiver noise figure, $E_{b}$ is the average energy per bit required for a given bit-error-rate (BER), and $R_{b}$ is the system bit rate. The receiver noise figure is $N_{f}$ and $N_{o}$ is the single-sided thermal noise PSD at room temperature [11].

The BER of an M-Array QAM, $2 R \times N$ MIMO system based on the Alamouti scheme $(M=2 b)$ with a square constellation (i.e. $b$ is even) in Rayleigh fading for $b \geq 2$ can be shown to be given by

$$
\begin{aligned}
\overline{P_{b}}=\frac{1}{2^{N_{T} N_{R}}}\left(1-\frac{1}{\sqrt{1+2 N_{o} / \overline{E_{b}}}}\right)^{N_{T} N_{R}} \\
\quad \times \sum_{k=0}^{N_{T} N_{R}-1} \frac{1}{2^{k}}\left(\begin{array}{c}
N_{T} N_{R}-1+k \\
k
\end{array}\right)(1 \\
\left.+\frac{1}{\sqrt{1+2 N_{o} / \overline{E_{b}}}}\right)^{k}, \quad \text { (8) }
\end{aligned}
$$

$P_{C}$ can be expressed as

$$
\begin{aligned}
P_{C} \approx N_{T}\left(P_{D A C}+\right. & \left.P_{\text {mix }}+P_{\text {filt }}\right)+2 P_{\text {synth }} \\
& +N_{R}\left(P_{L N A}+P_{\text {mix }}+P_{I F A}+P_{f i l r}\right. \\
& \left.+P_{A D C}\right)
\end{aligned}
$$

Where $P_{D A C}, P_{\text {mix }}, P_{\text {filt }}, P_{L N A}, P_{I F A}, P_{f i l r}, P_{A D C}, P_{\text {synth }}$ are the power consumption values for the digital-to-analog converter (DAC), the mixer, the low-noise amplifier (LNA), the intermediate frequency amplifier (IFA), the active filters at the transmitter side, the active filters at the receiver side, the analog-to-digital converter (ADC), and the frequency synthesizer, respectively. To estimate the values of $P_{D A C} \&$ $P_{A D C}$, we can use the model introduced in $[13,14]$. Finally, the total energy per bit for a fixed effective rate $R_{b}^{\text {eff }}$ can then be estimated as

$$
E_{p b}(d)=\frac{P_{P A}(d)+P_{C}}{R_{b}^{e f f}},
$$

\subsection{Virtual MIMO Communication}

In a wireless sensor network, the nodes often have small sizes. Thus, they are not being able to accommodate multiple antennas. Therefore, the implementation of MIMO based communication in a wireless sensor network requires sensor cooperation. In distributed wireless sensor networks, a set of low-end data collection sensors connected over a wireless link with a high-end data gathering node (DGN) that acts as a lead sensor. The energy resources of data collection sensor nodes are limited while the DGN has no energy constraints. The DGN is assumed not to have any energy constraint, or has relatively much longer battery life, and can be of larger physical sizes to accommodate multiple receiver antennas. This allows realization of true MIMO capability with only the transmitter side local communications [10].

In such wireless sensor networks, cooperative MIMO based communication can be achieved in two steps [9] the local communication and the long-haul communication. Assume a set of data collection nodes has data to be sent to the DGN. A 
set of nodes close to each other forms a cluster. Each node in the network associates itself with at least one cluster. The distance between nodes in a cluster is assumed to be considerably less than the distance between a cluster and the DGN. Energy consumption of the proposed cooperative MIMO-based scheme consists of two terms: the energy required for local communications among data collection sensors and the energy required for long-haul communications from data collection nodes to the data gathering node. To be consistent with earlier MIMO notation, we will assume that there are $N_{T}$ number of data collection sensors and the data gathering node is equipped with $N_{R}$ number of receiver antenna elements.

The energy consumption of DSC-MIMO focuses on the transmission of source data, which happens in intra cluster communication phase and inter cluster communication phase. Next, we use (9) to analyze the energy consumption of the two phases.

\subsubsection{Intra Cluster Communication}

The energy consumption for transmitting 1-bit original source data is

$$
\begin{aligned}
& E_{p b}^{M I M O}(d) \\
& =\frac{R_{b}^{\text {eff }}}{R_{b}}\left[\left.E_{p b}(d)\right|_{N_{T}=2, N_{R}=2}\right] R_{D S C},
\end{aligned}
$$

\subsubsection{Inter Cluster Communication}

In the inter cluster communication phase, every CG communicates with the next hop MCG through a $2 \times 2$ virtual MIMO link. Therefore, the energy consumption for transmitting 1-bit original source data is

$$
\begin{aligned}
& E_{p b}^{M I M O}(d) \\
& =\frac{R_{b}^{e f f}}{R_{b}}\left[\left.E_{p b}(d)\right|_{N_{T}=2, N_{R}=2}\right] R_{D S C}
\end{aligned}
$$

Where $R_{b}^{e f f}$ is the effective bit rate, which is given as

$$
R_{b}^{e f f}=\frac{F-p N_{T}}{F} R_{b}
$$

Where $F$ is the block size of space-time coding and $p$ is the Training overhead factor.

\subsection{Simulation Parameters}

Table 1: Simulation Parameter

\begin{tabular}{|c|l|}
\hline Wavelength $(\lambda)$ & $0.125 \mathrm{~m}$ \\
\hline Drain efficiency factor $(\alpha)$ & 0.47 \\
\hline$G_{t} G_{r}$ & $5 \mathrm{dBi}$ \\
\hline Noise power density $(\mathrm{No})$ & $-174 \mathrm{dBm} / \mathrm{Hz}$ \\
\hline Bandwidth (B) & $10 \mathrm{KHz}$ \\
\hline$P_{\text {mix }}$ & $30.3 \mathrm{~mW}$ \\
\hline$P_{I F A}$ & $20 \mathrm{~mW}$ \\
\hline$P_{\text {syn }}$ & $50 \mathrm{~mW}$ \\
\hline
\end{tabular}

\begin{tabular}{|c|l|}
\hline$P_{\text {filr }}=P_{\text {filt }}$ & $2.5 \mathrm{~mW}$ \\
\hline$P_{L N A}$ & $20 \mathrm{~mW}$ \\
\hline$N_{f}$ & $10 \mathrm{~dB}$ \\
\hline$M_{l}$ & $40 \mathrm{~dB}$ \\
\hline$P_{D A C}$ & $15.43 \mathrm{~mW}$ \\
\hline$P_{A D C}$ & $6.62 \mathrm{~mW}$ \\
\hline
\end{tabular}

\subsection{Simulation Analyses}

To verify the energy conservation effect of DSC-MIMO, we establish a simulation platform to compare the energy consumption with CMIMO in different communication phases. The main simulation parameters are shown in Table 1, which are the same as [7]. In addition, we set the rate of DSC as $75 \%$ which is the same as [15].

Figure 6 shows the communication energy consumption of DSC-MIMO and CMIMO in the intra cluster communication phase. To be fair, the control overhead of DSC-MIMO is counted to the energy consumption of intra cluster communication during the simulation.

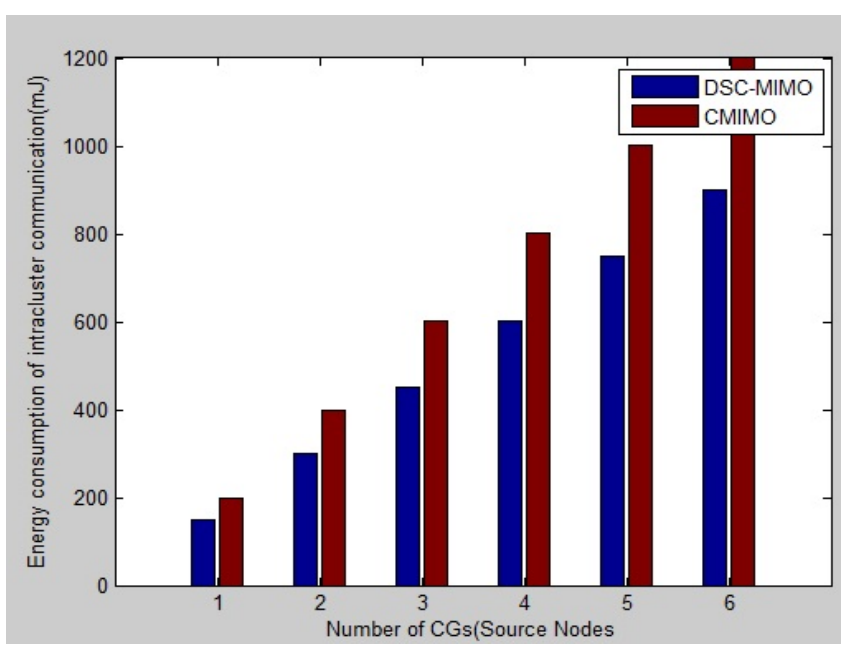

Fig.6 (a): Energy Consumption of DSC-MIMO and CMIMO in Intra cluster communication phase

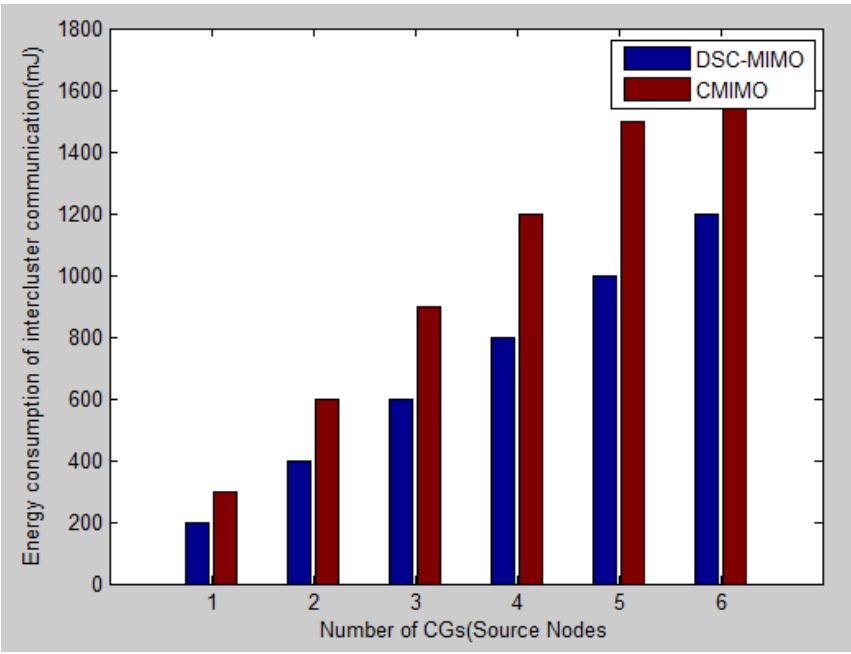

Fig.6 (b): Energy Consumption of DSC-MIMO and CMIMO in Inter cluster communication phase 
The result shows transmitting the same source data in intra cluster communication phase. The gap between DSC-MIMO and CMIMO is growing with the increasing of source nodes. There are two reasons causing this increasing gap. One is that DSC-MIMO improves the intra cluster communication process and makes the source data forwarded only once, while CMIMO has to forward source data twice. Although DSCMIMO increases some control overhead, the energy consumption of source data transmission is still the main part in this phase. The other is that the DSC is used in DSCMIMO which can compress the related source data and reduce the transmission data. Therefore, DSC-MIMO is more energy saving than CMIMO in intra cluster communication.

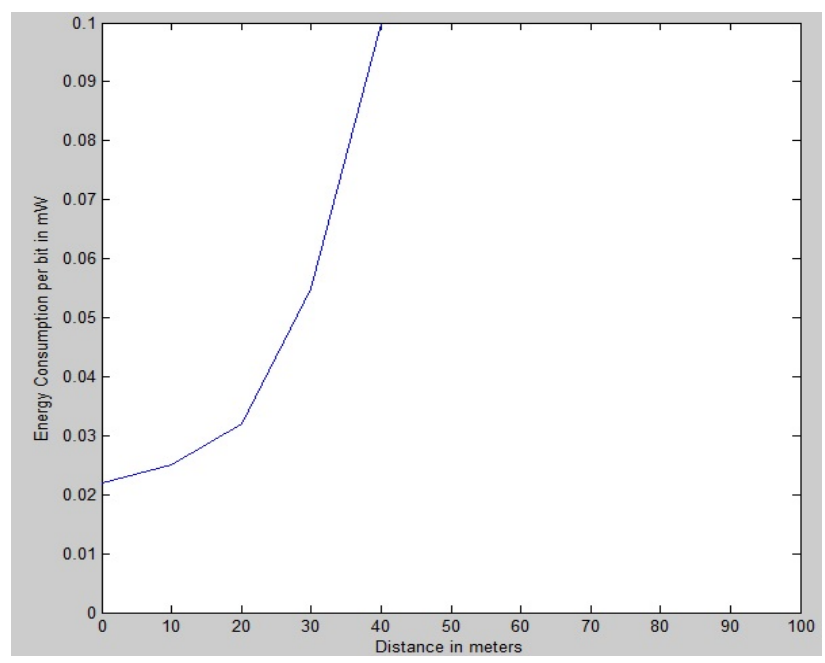

Fig.7 (a): Total energy consumption comparison of a system with a $2 \times 2$ CSISO based communication with path loss exponent of $k=2$.

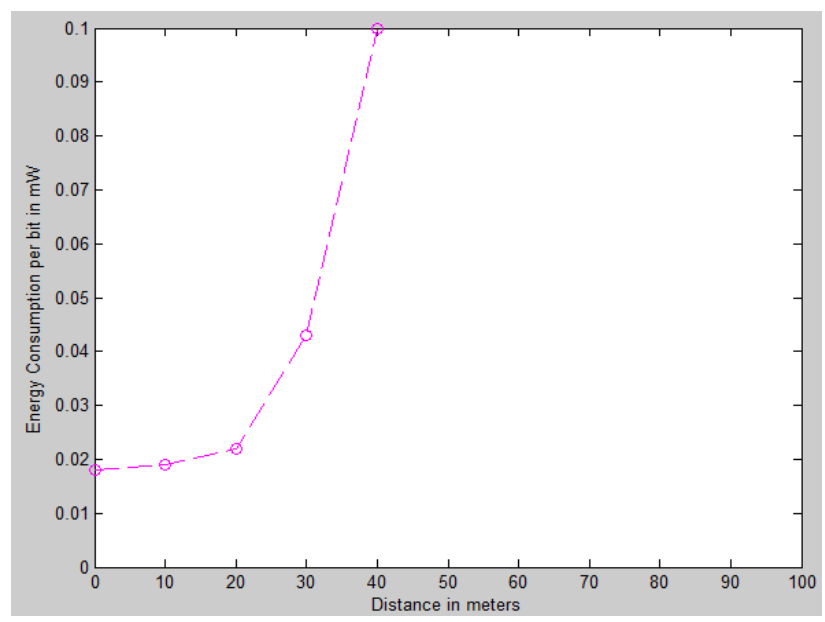

Fig.7 (b): Total energy consumption comparison of a system with a $2 \times 2$ DSC-SISO based communication with path loss exponent of $k=2$.

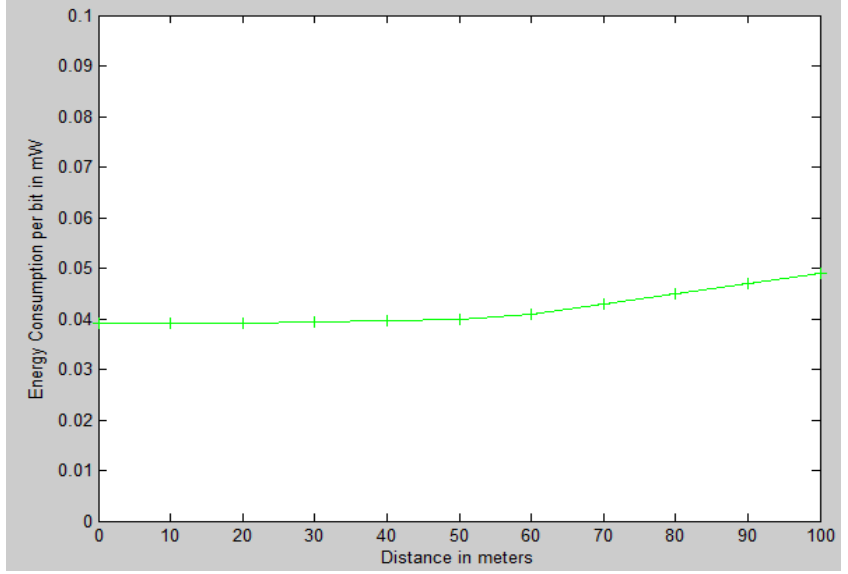

Fig.7 (c): Total energy consumption comparison of a system with a $2 \times 2$ CMIMO based communication with path loss exponent of $k=2$.

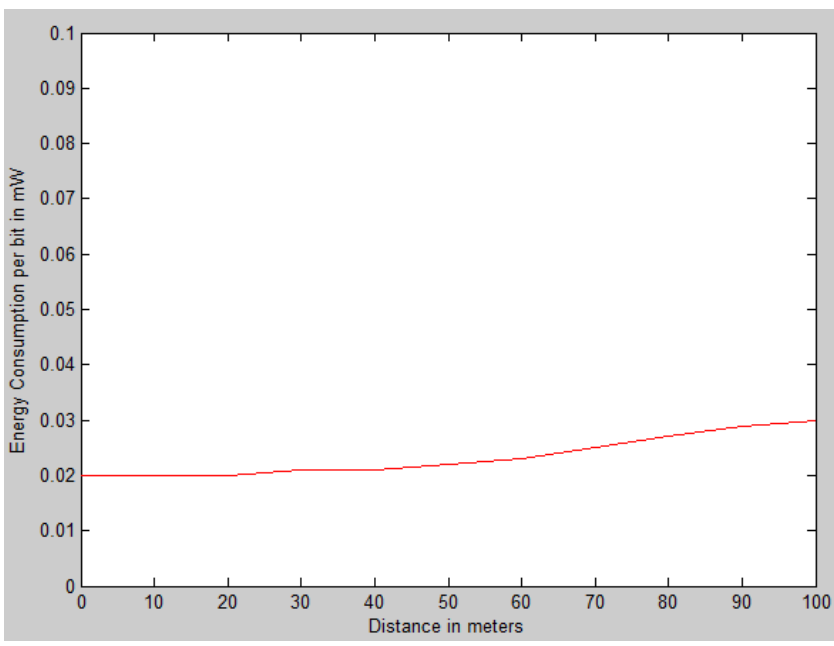

Fig.7 (d): Total energy consumption comparison of a system with a $2 \times 2$ DSC-MIMO based

communication with path loss exponent of $k=2$

Fig.7 shows the energy efficiency of DSC-MIMO and CMIMO in different transmission modes. We define the energy efficiency as the total energy consumption for transmitting 1-bit original source data. SISO is the transmission mode of intra cluster communication and MIMO is the transmission mode of inter cluster communication.

The simulation result shows that the SISO of DSC-MIMO is more efficient than CMIMO and the MIMO of DSC-MIMO is also more efficient than CMIMO. It is mainly because of the DSC in DSC-MIMO which compresses the related source data and reduces the length of transmission data. Therefore, the DSC can improve the energy efficiency of high node density WSN

\section{CONCLUSION}

Based on the existing virtual MIMO strategies for wireless sensor networks, we propose DSC-MIMO, which is very suitable for WSN with high node density and high scale, by improving intra cluster communication and importing the DSC. Different to the existing virtual MIMO communication strategies, DSC-MIMO uses cooperative groups constituted by source nodes to transmit the data directly and the process of source data collecting and forwarding by cluster heads is 
removed, which can reduce the forwarding times of source data and energy consumption.

The DSC can compress the related source data collected by adjacent nodes and improve the energy efficiency. The MCGs in DSC-MIMO can help WSN to realize the multi hop virtual MIMO transmission by receiving and forwarding the data from CGs of other clusters. CG is the most basic communication unit in DSC-MIMO, so how to divide CGs has a great impact on the effectiveness of communication in WSN. The cooperative group allocation algorithm proposed in this paper can easily select the best allocation scheme enabling WSN to achieve the best communication performance.

Finally, by comparing the energy efficiency of DSC-MIMO with CMIMO in simulation experiment, we prove that DSCMIMO is more energy-saving and has higher energy efficiency.

\section{REFERENCES}

[1] IEEE 802.15.4 standard for information technology. Part 15.4: Wireless Medium Access Control (MAC) and Physical Layer PHY Specification for Low-Rate Wireless Personal Area Networks (WPANS), 2006.

[2] Sivash M. Alamouti. A simple transmit diversity technique for wireless communications .IEEE Journal on Select Areas in Communications, 16(8): 1451-1458, October 1998.

[3] Wenqing Chen, Yong Yuan, Changchun Xu, and Kezhong Liuand Zongkai Yang. Virtual MIMO protocol based on clustering for wireless sensor network. Computers and Communications, 2005, ISCC 2005, Proceedings, 10th IEEE Symposium, 2005b.

[4] Shuguang Cui, Andrea J. Goldsmith, and Ahmad Bahai. Energy-efficiency of MIMO and cooperative MIMO techniques in sensor networks. IEEE Journal on Selected Areas in Communications, 22(6):1089-1098, August 2004.

[5] Shuguang Cui, Andrea J. Goldsmith, and Ahmad Bahai. Energy-constrained modulation optimization. IEEE Transaction on Wireless Communication, 4(5):2349 2360, Sept 2005

[6] Shuguang Cui, Andrea J. Goldsmith, and Ahmad Bahai. Cross-layer design of energy-constrained networks using cooperative mimo techniques. Invited for Publication at EURASIP'S Signal Processing Journal, August 2006.

[7] Ji-Huan $\mathrm{He}$ and $\mathrm{Xu}$-Hong Wu. Variatioanl iteration method: New development and applications. Computers and mathematics with applications, (54):881-894, 2007.

[8] Sudharaman K. Jayaweera. Energy analysis of MIMO techniques in wireless sensor networks. 38th Annual
Conf. on Information Sciences and Systems (CISS 04), Princeton,NJ, Mar 2004

[9] Sudharaman K. Jayaweera. Energy efficient virtual MIMO-based cooperative communications for wireless sensor networks. 2nd International Conf. on Intelligent Sensing and Information Processing and Information Processing (ICISIP'05, Jan 2005.

[10] Sudharaman K. Jayaweera. Virtual-MIMO based cooperative communication for energy-constrained wireless sensor networks. IEEE Transaction Wireless Communication, 5(5):984-989, May 2006.

[11] Sudharaman K. Jayaweera. An energy-efficient virtual MIMO communications architecture based on v-blast processing for distributed wireless sensor networks. IEEE Transaction Wireless Communication.

[12] Wenyu Liu and Mo Chen Xiaohua Li. Energy efficiency of MIMO transmissions in wireless sensor networks with diversity and multiplexing gains. Acoustic, Speech and Signal Processing, 4:897-900, March 2005.

[13] Wang Qing-hua, QU Yu-gui, LIN Zhi-ting, and BAI Rong-gang. Protocol for the application of co-operative MIMO based on clustering in sparse wireless sensor networks. The journal of China Universities of posts and telecommunications, 14(2), June 2007a.

[14] Wang Qing-hua, QU Yu-gui, LIN Zhi-ting, BAI Ronggang, ZHAO Bao-hua, and PAN Quan-ke. Protocol for the application of cooperative MIMO based on clustering in sparse wireless sensor networks. The journal of China universities of posts and telecommunications, 14(2), June 2007b.

[15] Yong Yuan and Zhihai He. Virtual MIMO-based crosslayer design for wireless sensor networks. Vehicular Technology, IEEE Transaction, 55(3), May 2006a.

[16] Yong Yuan and Zhihai He. A novel cluster-based cooperative MIMO scheme for multi-hop wireless sensor networks. EURASIP Journal on Wireless

[17] Communications and Networking, 2006(72493):1-9, 2006b. Yanbing Zhang and Huaiyu Dai. Energyefficiency and transmission strategy selection in cooperative wireless sensor networks. Journal of Communications and networks, 9(4), December 2007.

[18] S. Cui, A. J. Goldsmith, and A. Bahai, "Energyefficiency of MIMO and cooperative MIMO techniques in sensor networks," IEEE Journal on Selected Areas in Communications, vol. 22, no. 6, pp. 1089-1098, 2004.

[19] S. K. Jayaweera, "Virtual MIMO-based cooperative communication for energy-constrained wireless sensor networks," IEEE Transactions on Wireless Communications, vol.5,no. 5, pp. 984-989, 2006 